\title{
A novel PDX modeling strategy and its application in metabolomics study for malignant pleural mesothelioma
}

\author{
Zhongjian Chen ${ }^{1,2 \dagger}$, Chenxi Yang ${ }^{1,2 \dagger}$, Zhenying Guo ${ }^{1,2 \dagger}$, Siyu Song ${ }^{1,2}$, Yun Gao ${ }^{1,2}$, Ding Wang ${ }^{1,2}$,
} Weimin Mao ${ }^{1,2^{*}}$ and Junping Liu ${ }^{1,2^{*}}$

\begin{abstract}
Background: Malignant pleural mesothelioma (MPM) is a rare and aggressive carcinoma located in pleural cavity. Due to lack of effective diagnostic biomarkers and therapeutic targets in MPM, the prognosis is extremely poor. Because of difficulties in sample extraction, and the high rate of misdiagnosis, MPM is rarely studied. Therefore, novel modeling methodology is crucially needed to facilitate MPM research.

Methods: A novel patient-derived xenograft (PDX) modeling strategy was designed, which included preliminary screening of patients with pleural thickening using computerized tomography (CT) scan, further reviewing history of disease and imaging by a senior sonographer as well as histopathological analysis by a senior pathologist, and PDX model construction using ultrasound-guided pleural biopsy from MPM patients. Gas chromatography-mass spectrometry-based metabolomics was further utilized for investigating circulating metabolic features of the PDX models. Univariate and multivariate analysis, and pathway analysis were performed to explore the differential metabolites, enriched metabolism pathways and potential metabolic targets.
\end{abstract}

Results: After screening using our strategy, 5 out of 116 patients were confirmed to be MPM, and their specimens were used for modeling. Two PDX models were established successfully. Metabolomics analysis revealed significant metabolic shifts in PDX models, such as dysregulations in amino acid metabolism, TCA cycle and glycolysis, and nucleotide metabolism.

Conclusions: To sum up, we suggested a novel modeling strategy that may facilitate specimen availability for MM research, and by applying metabolomics in this model, several metabolic features were identified, whereas future studies with large sample size are needed.

Keywords: Patient-derived xenograft, Malignant pleural mesothelioma, GC-MS, Metabolomics, Ultrasound-guided biopsy

\footnotetext{
* Correspondence: liujunpingzjcc@163.com; maowm@zjcc.org.cn

${ }^{\dagger}$ Zhongjian Chen, Chenxi Yang and Zhenying Guo contributed equally to this work.

${ }^{1}$ The Cancer Research Institute, The Cancer Hospital of the University of Chinese Academy of Sciences (Zhejiang Cancer Hospital), Institute of Cancer and Basic Medicine (ICBM), Chinese Academy of Sciences, Zhejiang 310022, Hangzhou, China

Full list of author information is available at the end of the article
}

(c) The Author(s). 2021 Open Access This article is licensed under a Creative Commons Attribution 4.0 International License, which permits use, sharing, adaptation, distribution and reproduction in any medium or format, as long as you give appropriate credit to the original author(s) and the source, provide a link to the Creative Commons licence, and indicate if changes were made. The images or other third party material in this article are included in the article's Creative Commons licence, unless indicated otherwise in a credit line to the material. If material is not included in the article's Creative Commons licence and your intended use is not permitted by statutory regulation or exceeds the permitted use, you will need to obtain permission directly from the copyright holder. To view a copy of this licence, visit http://creativecommons.org/licenses/by/4.0/. The Creative Commons Public Domain Dedication waiver (http://creativecommons.org/publicdomain/zero/1.0/) applies to the data made available in this article, unless otherwise stated in a credit line to the data. 


\section{Introduction}

Malignant mesothelioma (MM) is a rare cancer originating from the mesothelial linings of the pleural or peritoneal cavities. Malignant pleural mesothelioma (MPM) is the predominant form of MM. The incidence of MPM is very low in China $(1.5 / 1000,000)$ [1]. Unfortunately, the prognosis of $\mathrm{MM}$ is extremely poor (survival time of 12-22 months) due to frequent late diagnosis that results from difficulties in early detection and lack in efficacy of current treatments [2]. Thus, MPM has been a dismal disease troubling both patients and clinical doctors.

MPM is normally induced by asbestos [3]. Although this mineral fiber has been widely banned globally, it is still consumed in several countries, such as India, Russia, and China [4]. Thus, MPM will continue to burden society due to previous and continuous use of asbestos as well as the long latency (20-40 years) of MPM [5]. However, most doctors and pathologists in China are unfamiliar with MM due to its rarity, leading to a delay in treatment and even misdiagnosis, denoting an urgent need in identification of diagnostic biomarkers [6].

Lack of specimens is the forefront obstacle in MPM research in China, even to the whole globe. Two studies suggested that the patient-derived xenograft (PDX) model, which implants tumor tissue from a patient into recipient mice, is suitable for testing both anti-cancer therapies and the biological functions of genes or proteins in MM $[7,8]$. Compared to cell line-derived xenograft, PDX model better simulates clinical samples, because it greatly reserves heterogeneity of the primary tumor [8]. Accordingly, PDX modeling is efficient and advantageous for biomarker detection and drug screening.

Construction of PDX models relies on specimens from patients, which are commonly collected from surgery. However, in China, surgery in MPM is infrequent thus can rarely provide samples for modeling. Ultrasoundguided (US-guided) biopsy is often used for examination of unclear malignancies, and it is still able to provide samples under situations when MPM patients are not suitable for surgery (e.g., pleural adhesions) [9]. Therefore, this study established a novel PDX modeling strategy based on an US-guided pleural biopsy, attributable to cooperation across three specializations, including US imaging, pathology, and lab research. Success in the establishment of two PDX models indicates the feasibility of our strategy.

To date, many studies focus on genomes in MM [1012], with relatively fewer investigating metabolomes. Nevertheless, metabolome has been viewed to utmost demonstrate phenotypes, indicating its importance and prominence for disease exploration through the discovery of metabolic biomarkers and therapeutic targets [13]. Bononi et al. reported that MM initiation is linked to metabolic reprogramming [14], and Zhang et al. also revealed that metabolic enzymes, such as SLC7A11, might serve as treatment targets for MM [15]. For the above reasons, we aimed to detect diagnostic metabolites and discover biological targets for MPM, through serumbased metabolomic profiling for the US-guided pleural biopsy-derived PDX model.

\section{Materials and methods}

\section{Ethical statement}

This study was performed in accordance with the Declaration of Helsinki (revised in 2013), and the protocol was approved by The Ethical Committee of Zhejiang Cancer Hospital (approval number: IRB-2018-9). Informed consent was obtained from each patient.

\section{Patients screening for PDX construction}

Patients who displayed pleural thickening in computed tomography (CT) imaging were reviewed by an experienced sonographer (Junping Liu) between March $1^{\text {st }}$ 2018 and December 30 2019 in Zhejiang Cancer Hospital. After exclusion, US-guided pleural biopsy was performed for the remaining patients. Those from patients who did not meet the following exclusion criteria were highly suspected (by the sonographer based on his past clinical experiences) to be MM: 1) pleural metastasis from lung cancer; 2) pleural metastasis from other cancers; 3) tuberculosis lesions; 4) unclear diagnosis; and 5) other reasons (according to imaging features and clinical history). Under the premise of not interfering with clinical diagnosis, these biopsies were collected and implanted into immunodeficient mice for constructing PDX models. At the same time, all biopsies were sent to a senior pathologist (Zhenying Guo) for precise diagnosis.

The animal experiment was permitted by the Institutional Animal Care and Ethics Committee of Zhejiang Cancer Hospital (No. 2018-03-054), in compliance with national or institutional guidelines for the care and use of animals. All the animals were euthanized using isoflurane at the end of experiment.

\section{US-guided pleural biopsy}

Patients were fasted for at least $8 \mathrm{~h}$ before biopsy. The biopsy was performed according to Zhang et al. [16] Briefly, Esaote MylabTMTwice ultrasound apparatus (Italy) with probes configuration convex transducer CA541 and linear LA524 was utilized. First, a lowfrequency probe $(4.0 \mathrm{MHz})$ was used to detect the pleural effusion, pleura and blood flow, and the thickest point of the pleura was selected for biopsy. Second, pleural biopsy was guided through alternatively using low-frequency $(4.0 \mathrm{MHz})$ and high-frequency $(9.0 \mathrm{MHz})$ probes conducted by two experienced sonographers. One sonographer provided the guidance, and the other 
used an 18 or $16 \mathrm{G}$ automated cutting needle (MC1816, Bard Max. Core, Bard Inc., USA) to perform the biopsy under local anesthesia with $2 \%$ lidocaine. Specimens were immediately fixed in $10 \%$ formalin and sent to the Department of Pathology for examination. Without interfering clinical diagnosis, part of fresh specimens was sent for constructing PDX models.

\section{Immunohistochemistry (IHC) staining}

IHC staining was performed according to Wu et al. [8] In brief, slides of formalin-fixed and paraffin-embedded tumors were deparaffinized and incubated in $3 \%$ hydrogen peroxidase. After inactivation of endogenous peroxidases, slices were treated with antigen retrieval by boiling at $100{ }^{\circ} \mathrm{C}$ for $90 \mathrm{~s}$ in citric acid repair solution $(\mathrm{pH}=6)$. After blocking, slices were incubated with antibodies overnight at $4{ }^{\circ} \mathrm{C}$. Slices were then incubated with HRP-labeled secondary antibody for $30 \mathrm{~min}$. According to gender, histological features, and location of the tumor, a panel of biomarkers for IHC analysis was selected and the diagnosis was made by a senior pathologist (Zhenying Guo). Details of the antibodies used were listed in the supplemental information.

\section{PDX model construction}

The procedure of PDX construction was similar to established protocol from Wu et al. [8] In brief, 5-weekold female BALB/c immunodeficient mice (certificate number: 2017005004641) were purchased from Shanghai Slac Laboratory Animal Company (Shanghai, China). The mice adapted to the environment for 1 week. Every five of all mice were kept in one cage with free access to food and water, to a $12 \mathrm{~h} / 12 \mathrm{~h}$ light/dark cycle, at temperature between $22{ }^{\circ} \mathrm{C}$ and $26^{\circ} \mathrm{C}$, with $55 \%$ relative humidity. Fresh tumor tissue was kept in a sterilized PBS buffer on ice, and was cut into blocks of $2 \times 2 \times 2$ $\mathrm{mm}$ and then was engrafted subcutaneously into the flanks of BALB/c mice (P0) with a trocar needle. A PDX model which can be consecutively passed over twice is defined as a success (P2). Then P2 tumors were harvested and transplanted to 10 mice for the PDX model, and 7 mice without treatment were taken as a control group. When the average tumor size exceeded $200 \mathrm{~mm}^{3}$, blood was collected retro-orbitally under isoflurane anesthesia. Sera were separated with centrifugation for 10 min at $2400 \mathrm{~g}, 4^{\circ} \mathrm{C}$ and were kept at $-80^{\circ} \mathrm{C}$ until analysis.

\section{Gas chromatography-mass spectrometry (GC-MS)-based metabolomics}

The GC-MS-based metabolomics was performed according to the previously published method from Zhao et al. [17] The procedures including sample preparation and GC-MS analysis are described in the supplemental information.

\section{Metabolomic data analysis}

After data formatting (to .abf) with Reifycs Abf Converter (https://www.reifycs.com/AbfConverter/), MSDIAL software was used for data processing, including peak picking, peak alignment, missing value interpolation, and so on. Metabolite annotation was performed through the untargeted database of GC-MS from Lumingbio. Finally, a data set with sample information and peak information was obtained. Principal component analysis (PCA) and partial least-squares-discriminant analysis (PLS-DA) were performed to visualize the metabolic shift among groups using R package ropls (version 1.18.8). Variable importance in the projection (VIP) was obtained from PLSDA, which ranks the contribution of metabolite features in the PLS-DA model. Finally, features with VIP $>1.0$ and $P$-value $<0.05$ from two-tailed Student's t-test were defined as differential metabolites. Heatmaps were plotted to illustrate the metabolic patterns using $\mathrm{R}$ package pheatmap (version1.0.12). Metabolic pathway analysis was performed using the online tool Metaboanalyst 5.0 (https://www.metaboanalyst.ca/MetaboAnalyst), and results were visualized using $\mathrm{R}$ package ggplot2 (version 3.3.3). Illustrations of metabolites and their corresponding pathways were created with BioRender (https://biorender.com).

\section{Results}

\section{Patient information and model construction}

From March $1^{\text {st }} 2018$ to December $30^{\text {th }} 2019$, 158 patients with pleural thickening were reviewed by a senior sonographer (Junping Liu) (Fig. 1). A typical pleural thickening in CT scan was shown in Fig. 2A. A total of 42 individuals were excluded, and the remaining $(n=$ 116) patients were performed with US-guided pleural biopsy and pathological diagnosis (Fig. 1, Fig. 2B, Table S1). Fourteen of them were suspected to be MPM (Table S2), among which only 10 biopsies were accessible and were subsequently implanted into mice. Biopsies pathologically diagnosed with other diseases $(n=5)$, including lung adenocarcinoma $(n=3)$ and rhabdomyosarcoma $(n=2)$, were further excluded (Fig. 1). Among the 5 pathologically confirmed MM, 3 failed to grow on mice from P0 to P2, while 2 were successfully constructed. PDX1 was from a 55-year-old male with pleural thickening of annular nodules, lumps in the lungs and chest wall; while PDX2 was from an 85-year-old female, who showed pleural thickening of annular nodules, chest wall lumps. Their detailed information was shown in Table 1.

\section{PDX model confirmation by IHC}

In the metabolomics study, 6 out of 10 PDX1 models (P3) and 8 out of 10 PDX2 (P3) model grew and were used for GC-MS based metabolomics. Figures 3 and 4 


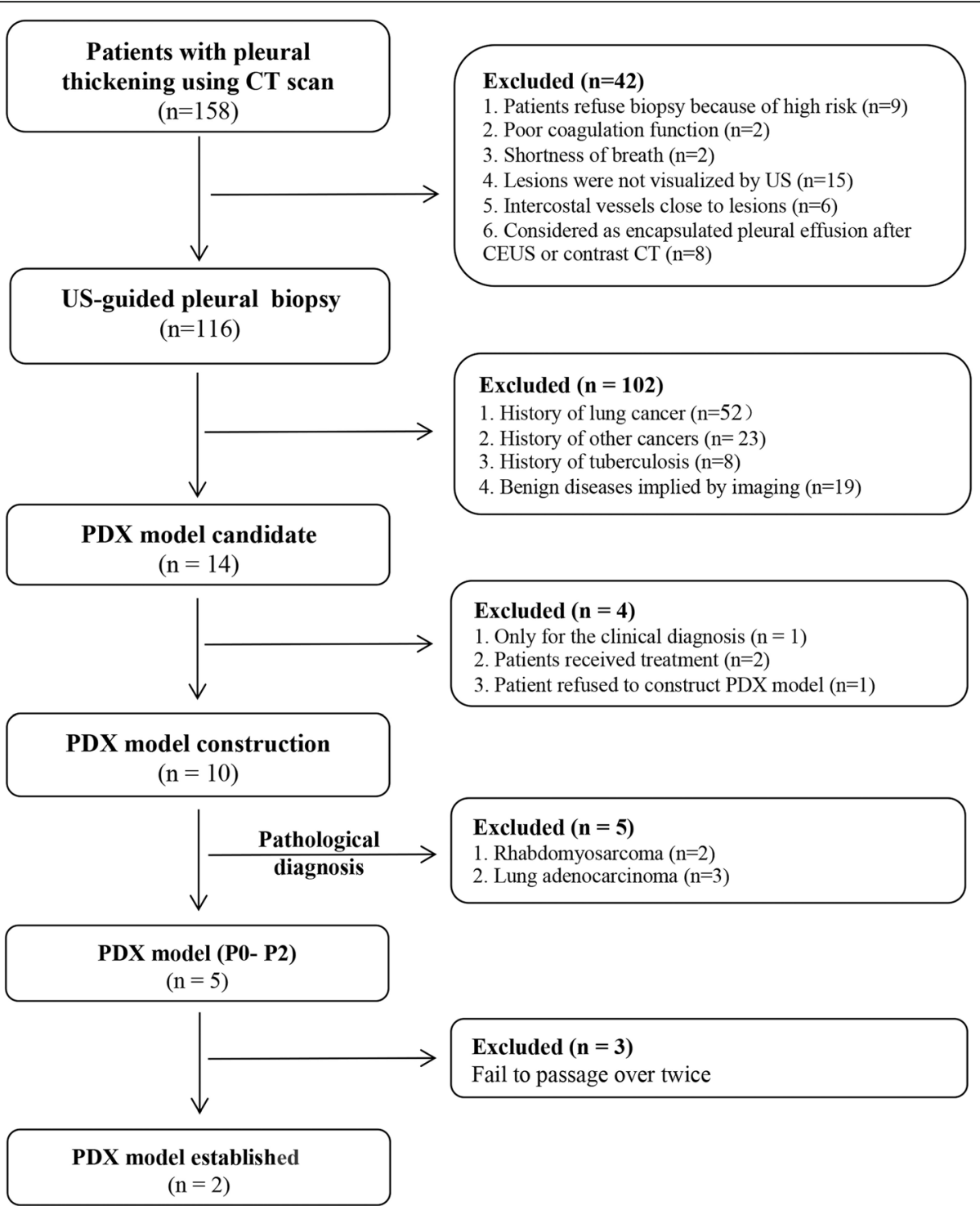

Fig. 1 Ultrasound-guided pleural biopsy-based PDX modelling strategy for malignant pleural mesothelioma

demonstrated Haemotoxylin and Eosin stains (HE) and IHC results of the two primary tumors and the xenograft tumors, PDX1 (Fig. 3) and PDX2 (Fig. 4). HE stains presented the epithelioid (Fig. 3A, G) and sarcomatoid (Fig. $4 \mathrm{~A}, \mathrm{H})$ features of MPM for the primary tumors and PDX1\&2 tumors. Positive expression of CR, CK5/6, WT1, and D2-40 (Fig. 3B-E) were denoted in primary epithelioid tumor tissue, and PDX1 expressed the same pattern (Fig. 3H-K). TTF1 negative in primary tumor (Fig. 3F) and PAX8 negative in PDX1 (Fig. 3L) distinguish MPM from metastatic lung adenocarcinomas. Altogether, these results confirmed the pathological subtype of epithelioid mesothelioma, and suggested successful construction of a reliable PDX model.
Similarly, expression of CR (Fig. 4B), and WT1 (Fig. 4C) confirmed the nature of the second model tumor being mesothelioma. CAM5.2 positive (Fig. 4E) distinguished this tumor from sarcoma. But CK5/6 (Fig. 4D) negative and VIM positive (Fig. 4F) can still discriminate its sarcomatoid identity from the epithelioid mesothelioma. In accordance, immunoprofiling for PDX2 showed CR (Fig. 4I), WT1 (Fig. 4J), and CAM5.2 (Fig. 4K) positive. In addition, DES (Fig. 4G) negative of primary tumor of PDX2 indicated poor differentiation ability of this tumor.

Serum metabolic shift between PDX models and controls In total, 209 metabolites in serum samples were annotated. After multivariate analyses, samples in PDX1 

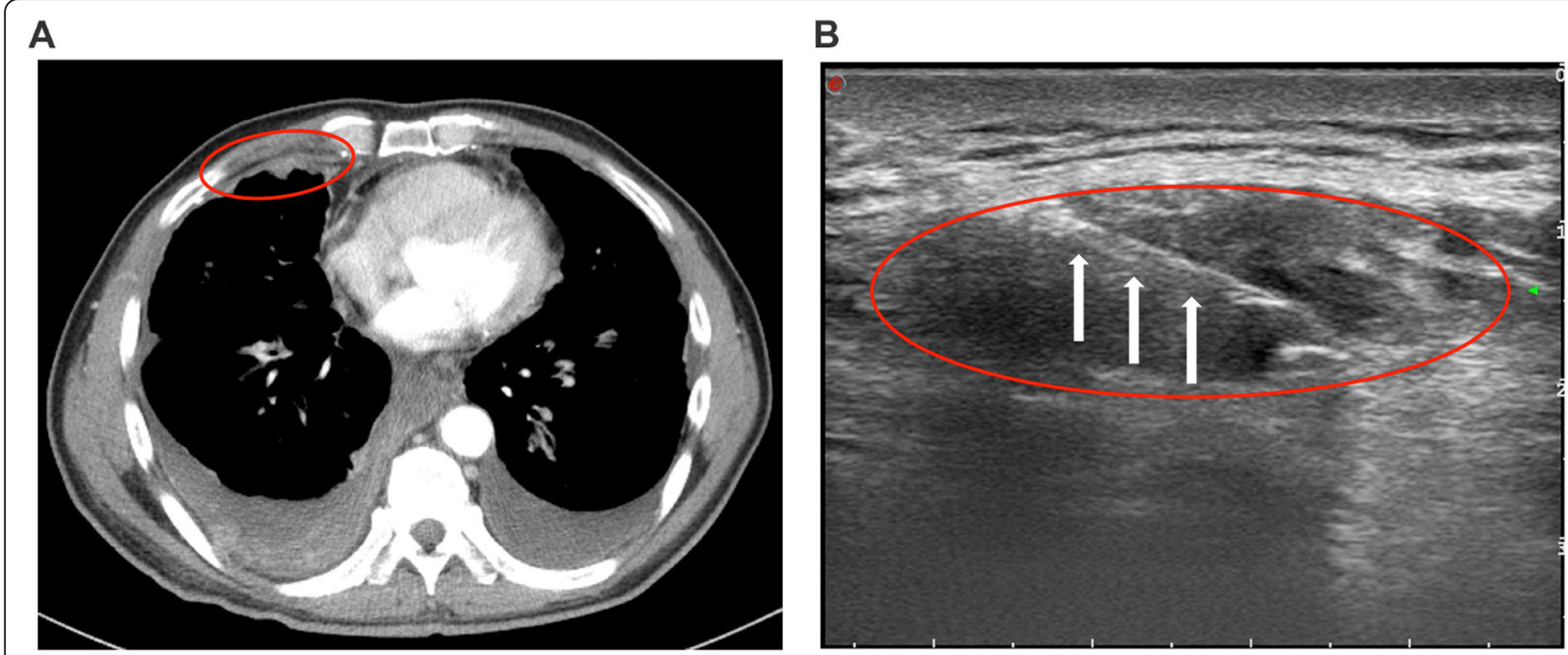

Fig. 2 Typical CT scan and ultrasound imaging of patients with pleural thickening. (A) CT scan, red oval refers to the pleural thickening area; (B) Ultrasound imaging, red oval refers to the pleural mass, white arrows point to the biopsy needle

group were successfully separated from control group by unsupervised PCA (Fig. S1A), whereas PDX2 group cannot be separated from control group (Fig. S1B). In supervised PLS-DA models, clearer separation trend for PDX 1 (Fig. S1C) and 2 (Fig. S1D) were present, though the latter remains ambiguous. Volcano plots revealed significant changes (VIP $>1.0$, $P$-value $<0.05)$ in metabolites in between PDX1 and 2 (Fig. S2), and each in comparison to controls (Fig. S1E, F). In PDX1 versus controls, 58 upregulated metabolites and 23 downregulated metabolites were obtained. And in PDX2, 21 upregulated metabolites and 3 downregulated metabolites were obtained. In addition, of the 12 overlapped metabolites, 10 were upregulated and 2 were downregulated, in both PDX1 and PDX2 versus controls. Between PDX1 and PDX2, 10 upregulated and 14 downregulated metabolites were presented. Detailed information of differential metabolites (including VIP, $P$-value, and fold change) was listed in Table S3 (PDX1 vs. control), Table S4 (PDX2 vs. control), Table S5 (overlapped in PDX1 and PDX2 compared to controls), and Table S6 (PDX1 vs. PDX2).

Table 1 Patient characteristics for PDX1 and PDX2 models

\begin{tabular}{lll}
\hline Parameters & PDX1 & PDX2 \\
\hline Sex & Male & Female \\
Age (year) & 55 & 85 \\
Body mass index & 20 & 15 \\
Asbestos exposure & No & No \\
Smoking & No & No \\
Drinking & Yes & No \\
Tumor location & Pleural & Pleural \\
Cell types & Epithelioid & Sarcomatoid \\
CT scan & Pleural thickening of annular nodules, & Pleural thickening of annular nodules, chest wall lumps \\
& lumps in the lungs and chest wall & \\
Family history of & No & Yes \\
tumor & & \\
Immunohistochemistry & CK(+), Ki-67(+,70\%), TTF1(-), CK5/6(+), WT- & TTF1(-), CK(+), EMA(+), Vim(+), CRICalretinin(+), D2-40(-), CK5/6(-), WT-1(-), \\
& 1(+), CRICalretinin(+) & NapsinA(-), P63(-), MyoD1(-), Myogenin(-), S-100(+), CAM5.2(+),SOX10(-), \\
& & HMB45(-), Melan-A(-), CD31(+), CD34(-), SMA(+), Des(-), Ki-67(+,60\%) \\
Somatic mutation & BAP1(p.Glu200fs), PIK3CA(p.Glu545Lys), & NA \\
& PTEN(p.Arg130*), TP53(p.Arg273His) & \\
\hline
\end{tabular}



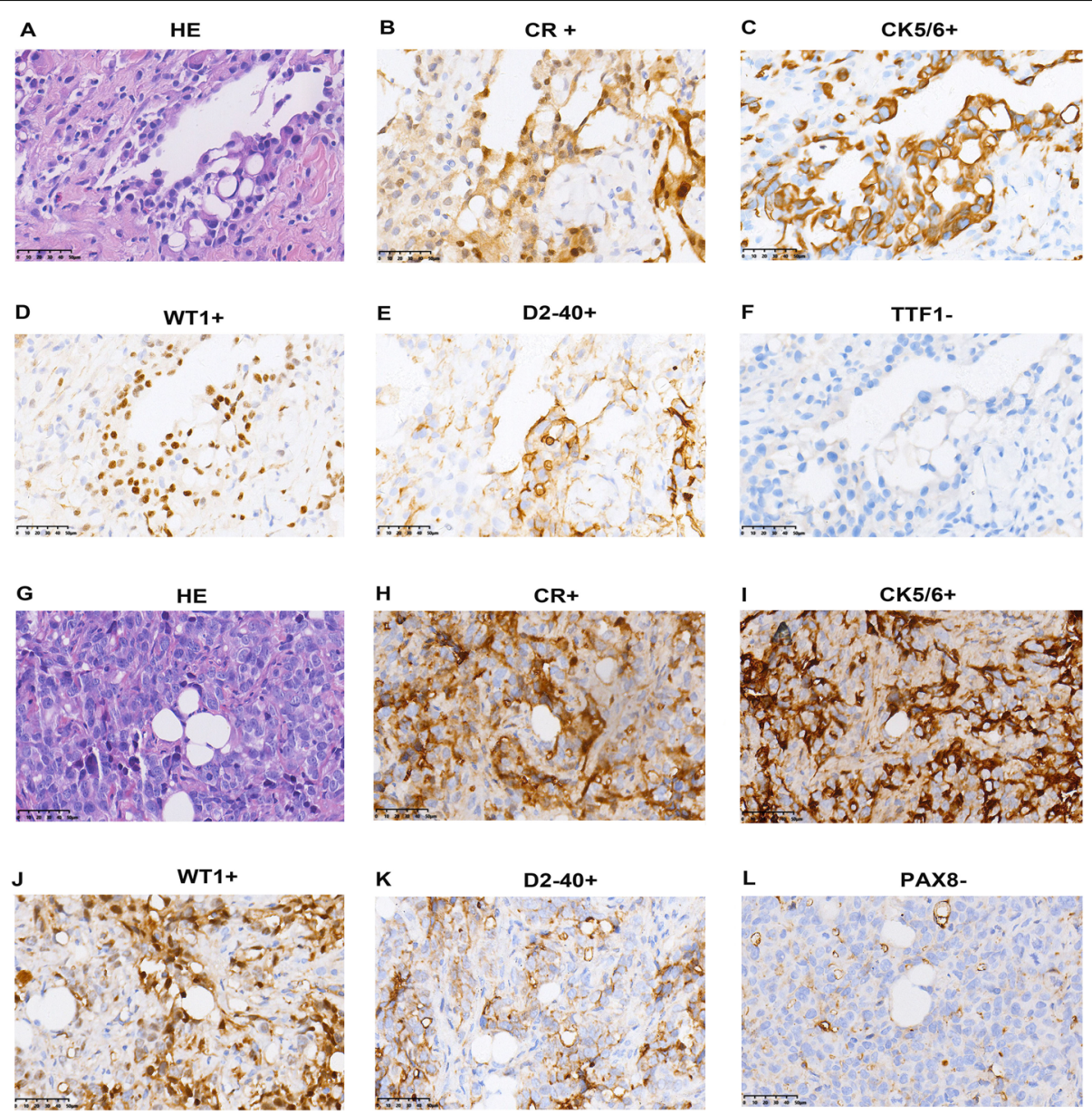

Fig. $3 \mathrm{HE}$ staining and IHC staining of tumor tissue from the original patient and PDX1 model (P3). For original tumor: (A) HE staining, (B-E) CR, CK5/6, WT1, and D2-40 positive (F) TTF1 negative; for PDX1's tumor: (G) HE staining, (H-K) CR, CK5/6, WT1, and D2-40 positive, (L) PAX8 negative (original magnification $\times 400$ )

\section{Metabolic patterns and pathway enrichment}

Hierarchical clustering heatmaps were then conducted and showed distinctive differentially expressed metabolic patterns between PDX1 and control $(n=82$; Fig. 5), PDX2 and control ( $n=25$; Fig. 6), overlapped between PDX1 and $2(n=12$; Fig. 7A), and between PDX1 and PDX2 ( $\mathrm{n}=25$; Fig. 7B). Figure 8 illustrated enriched pathways of the differential metabolites between PDX1 vs. control (Fig. 8A, Table S7), PDX2 vs. control (Fig. 8B, Table S8), overlapped in PDX1 and PDX2 (Fig. 8C, Table S9), and PDX1 vs. PDX2 (Fig. $8 D$, Table S10). Figure 9 showed all annotated metabolites presented and their enrichments in amino acid metabolism, TCA cycle and glycolysis, and nucleotide metabolism in PDX1, PDX2, and control. With the knowledge of the enriched pathways and the metabolites involved, an overall illustration of amino acid metabolism, tryptophan degradation, glycolysis, and TCA cycle was pictured in Fig. 10.

\section{Dysregulated amino acid metabolism in MPM}

Among the 81 significant dysregulated metabolites presented in PDX1, 5 were amino acids with 4 downregulated and 1 upregulated. Within the 5 amino acids, upregulated kynurenine and downregulated serotonin were involved in tryptophan degradation. Meanwhile, 4 of top 10 most significantly enriched pathways in PDX1 were amino acid metabolism pathways, and according to rich factor and $P$ value, the aspartate and glutamine metabolism; and arginine biosynthesis were the top 2 enriched pathways.

In PDX2, no significant changes were observed in amino acids. Nevertheless, similar to PDX1, kynurenine was also significantly upregulated, with a fold change of 1.99 compared to controls. However, tryptophan itself 


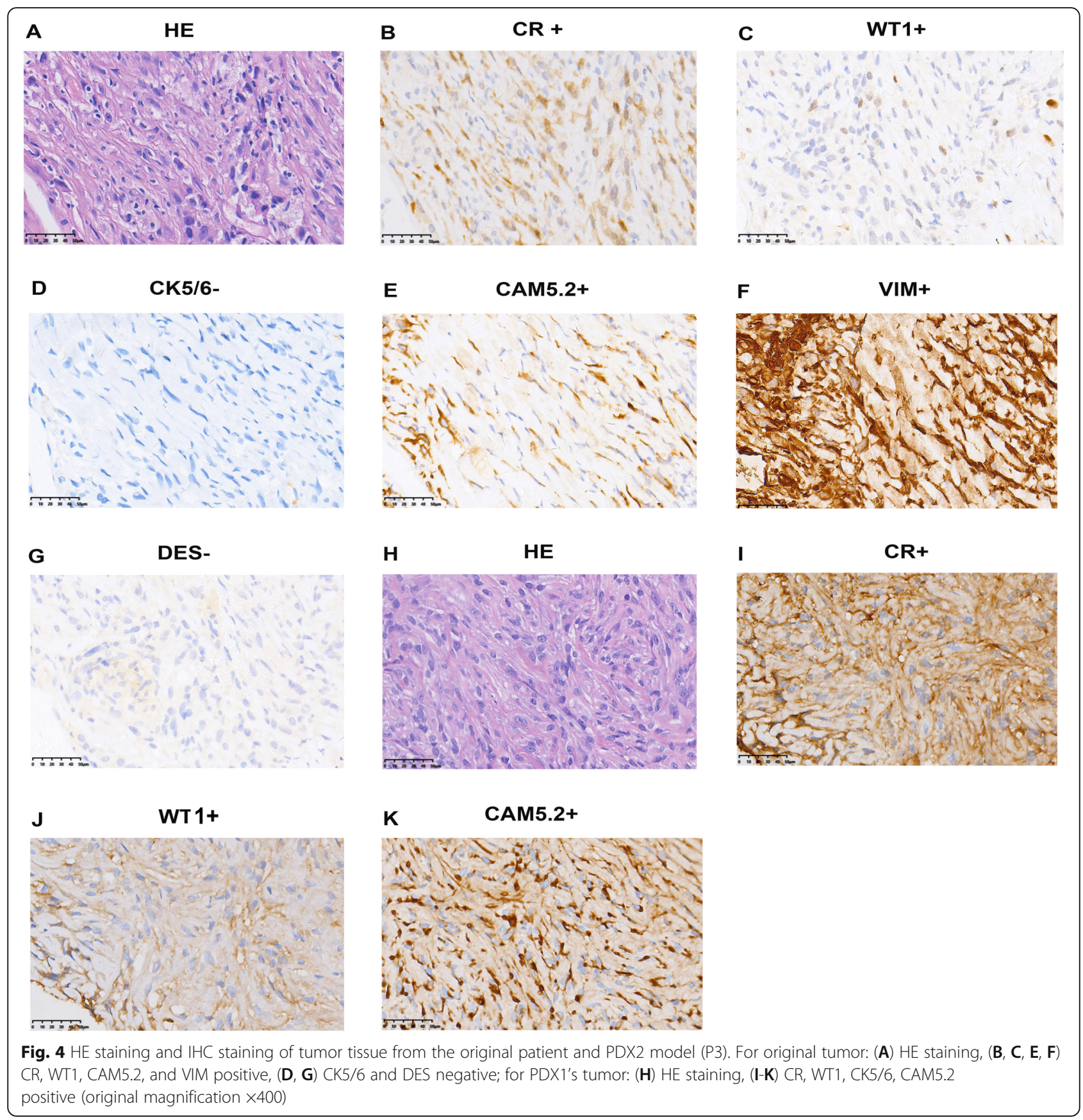

did not display significant change in circulating levels in both PDX models.

\section{Dysregulated glycolysis and TCA metabolites in MPM}

The top 3 most significantly enriched pathways in PDX2 were citrate cycle, pyruvate metabolism, and glycolysis/ gluconeogenesis. In line, two metabolites in pyruvate metabolism, bisphosphoglycerol and pyruvic acid, were all upregulated, with fold changes of 1.46 and 1.37, respectively. Additionally, the end product of Warburg aerobic glycolysis, lactic acid, was found upregulated in PDX2 but not in PDX1.

Succinic acid and pyruvic acid are overlapped metabolites of TCA cycle in both PDX models that showed significant changes. Succinic acid increased 2.17 folds in PDX1 and 1.49 folds in PDX2; and pyruvic acid increased 1.40 folds in PDX1 and 1.37 folds in PDX2. In addition, in PDX1, glutamine was downregulated to 0.49 folds, whereas fumaric acid was upregulated to 1.82 folds. But no significant alterations in other TCA compartments were detected in PDX2. 


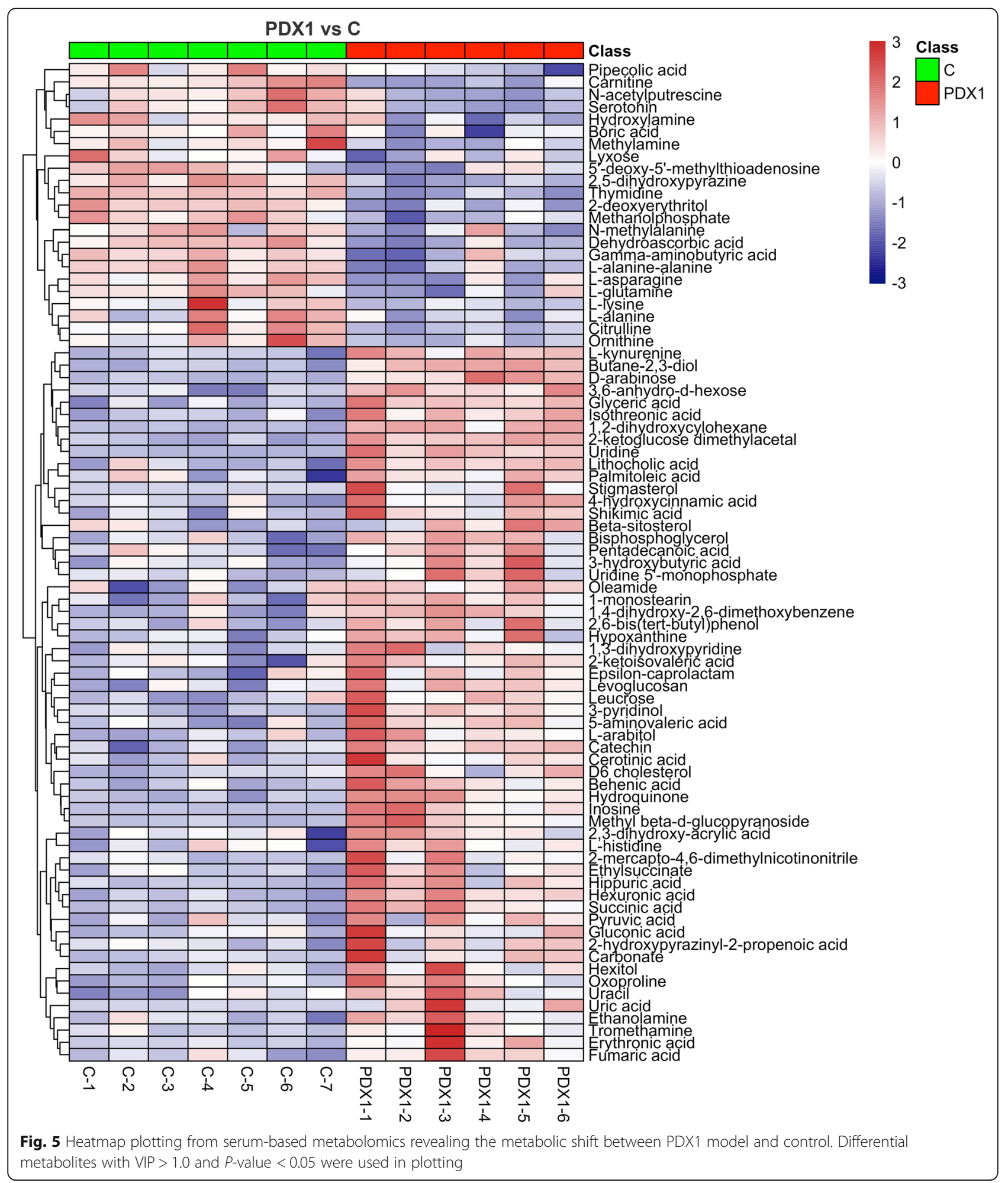

\section{Abnormality in nucleotide metabolism}

We detected uridine 5'-monophosphate (UMP), uridine, thymidine, and uracil enrichment in pyrimidine metabolism. In addition, hypoxanthine, inosine, allantoate, uric acid, and urea were enriched in purine metabolism. Consistently, uric acid, uridine, and inosine were significantly upregulated in both PDX1 and PDX2. Uracil, UMP, and hypoxanthine were upregulated in both 


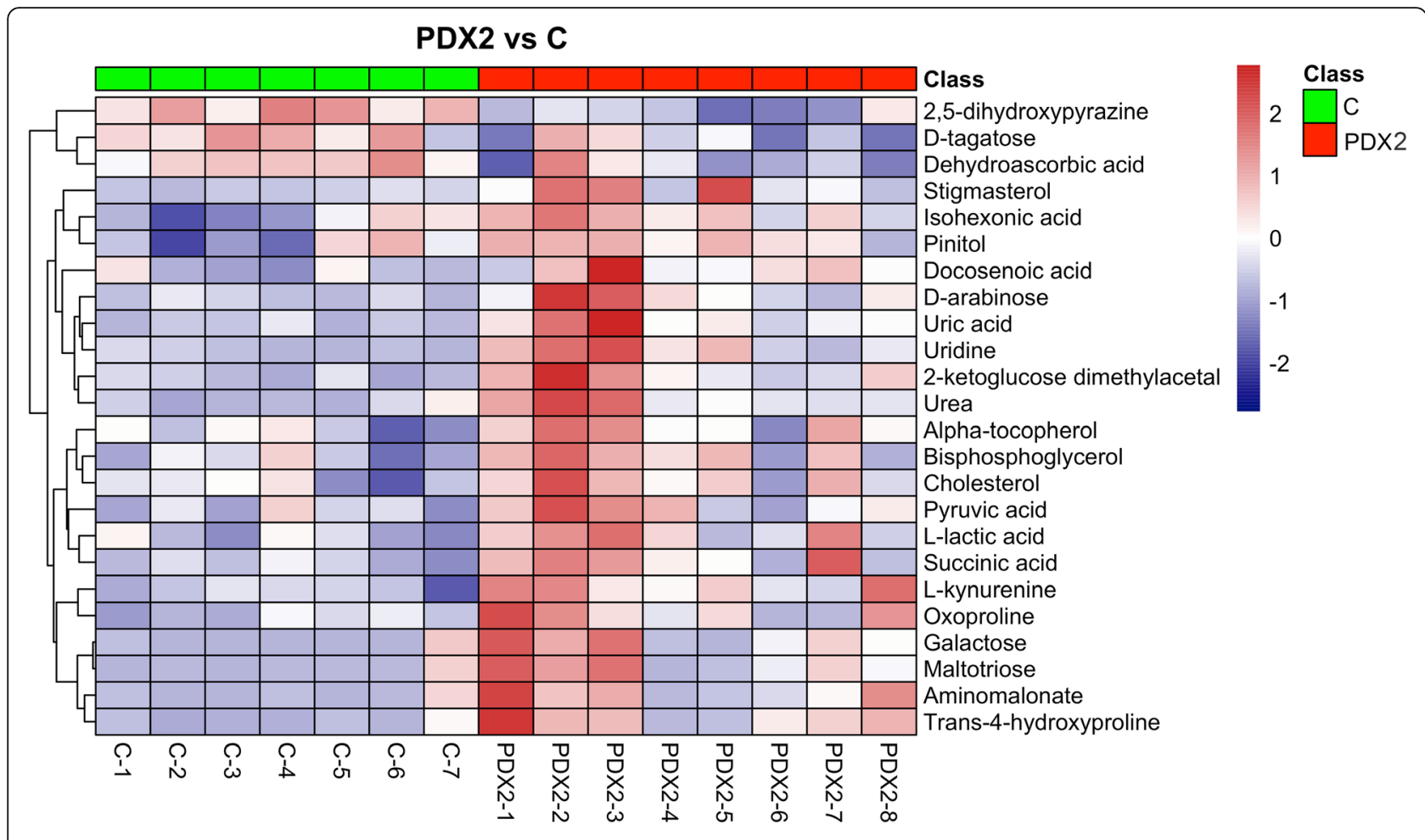

Fig. 6 Heatmap plot for comparison between PDX2 and control. Differential metabolites with VIP $>1.0$ and $P$-value $<0.05$ were used in plotting

models, whereas the trends were only significant in PDX1. Urea was only significantly increased in PDX2 with a fold change of 1.40 , while thymidine was only downregulated in PDX1 with a fold change of 0.40. No change was detected in levels of allantoate in both models.

\section{Discussion}

Sample scarcity has been an issue for research in MPM. Our methodology for PDX modeling from US-guided pleural biopsy in part removes this restraint, as biopsy samples are more accessible than samples from surgery. Although biopsy during video-assisted thoracoscopic surgery (VATS) was the gold standard for MPM diagnosis in current, it is more invasive than US-guided biopsy. Notably, dedicated examination and sampling used in our strategy helped improve efficiency as well as reduce the cost in modeling. For pleural lesions, a US-guided pleural biopsy was utilized, which outstands for its realtime multiplanar visualization that aids sampling [18]. Then the senior sonographer helped narrow specimens down to MPM candidates for modeling through the aforementioned exclusion criteria. Precise diagnosis by an experienced pathologist then helped rule out nonMPM PDXs. Thereby, our modeling strategy can diminish the waste of resources and time while retaining the reliability of PDX models.
In addition, PDX models offer a more manipulative environment for developing therapies and detecting biomarkers compared to human beings [19], as well as better retain heterogeneity of tumor compared to cell lines [8]. Many cancer studies use PDX model as it highly resembles the primary tumor implanted into it [20-23]. Meanwhile, such model parallels the original tumor better than cell lines, and concurrent results are closely relevant to the clinic [8]. Hence, for the above reasons, this modeling strategy is insightful and feasible, which can facilitate not only MPM-related research but also the research of other rare cancers limited by lack of specimens.

By further applying metabolomics based on PDX sera, we detected a panel of dysregulated metabolites and enriched pathways. A significant proportion of metabolic changes denoted in our results was verified in other clinical studies, indicating the reliability of both our metabolomics method and the PDX model. Therefore, we encourage the use of US-guided pleural biopsy for PDX modeling in combination with metabolomics for investigation in rare cancers, including MPM, which brings an opportunity for the identification of predictive markers and treatment targets.

Our metabolomics results revealed a dysregulated amino acid metabolism in MPM. Many studies had reported that cancers vastly demand amino acid for ATP 
A

Overlapped metabolites between PDX1 and PDX2

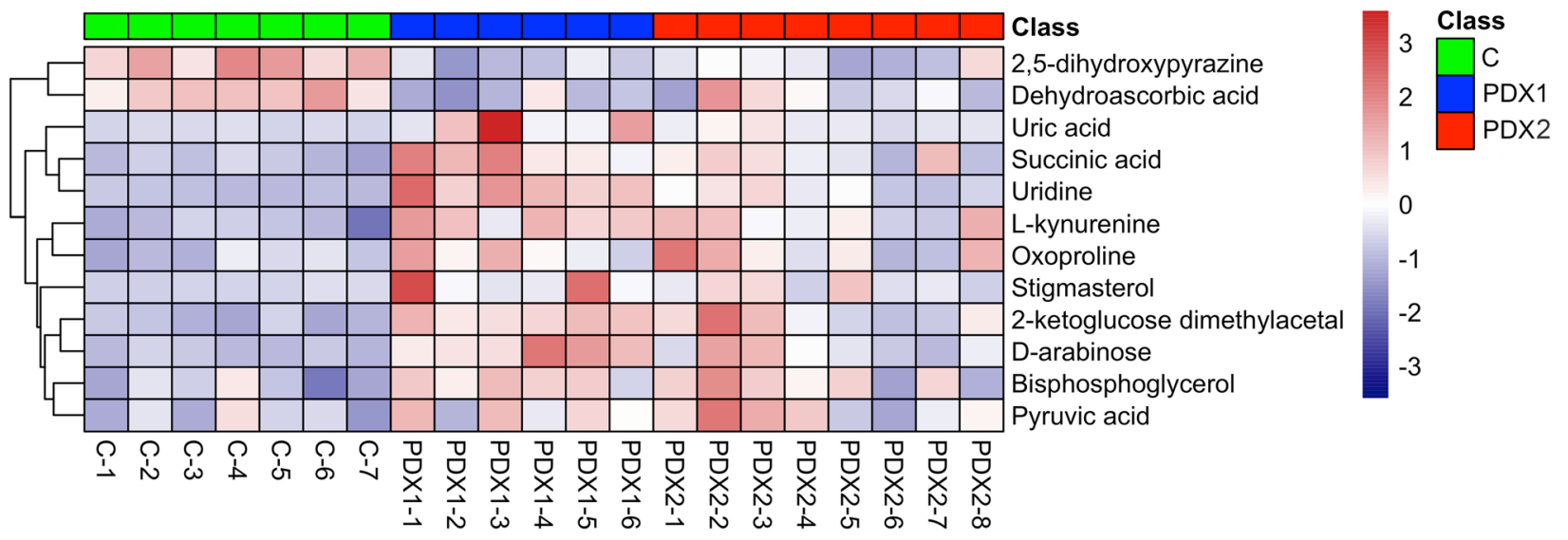

B

PDX1 vs PDX2

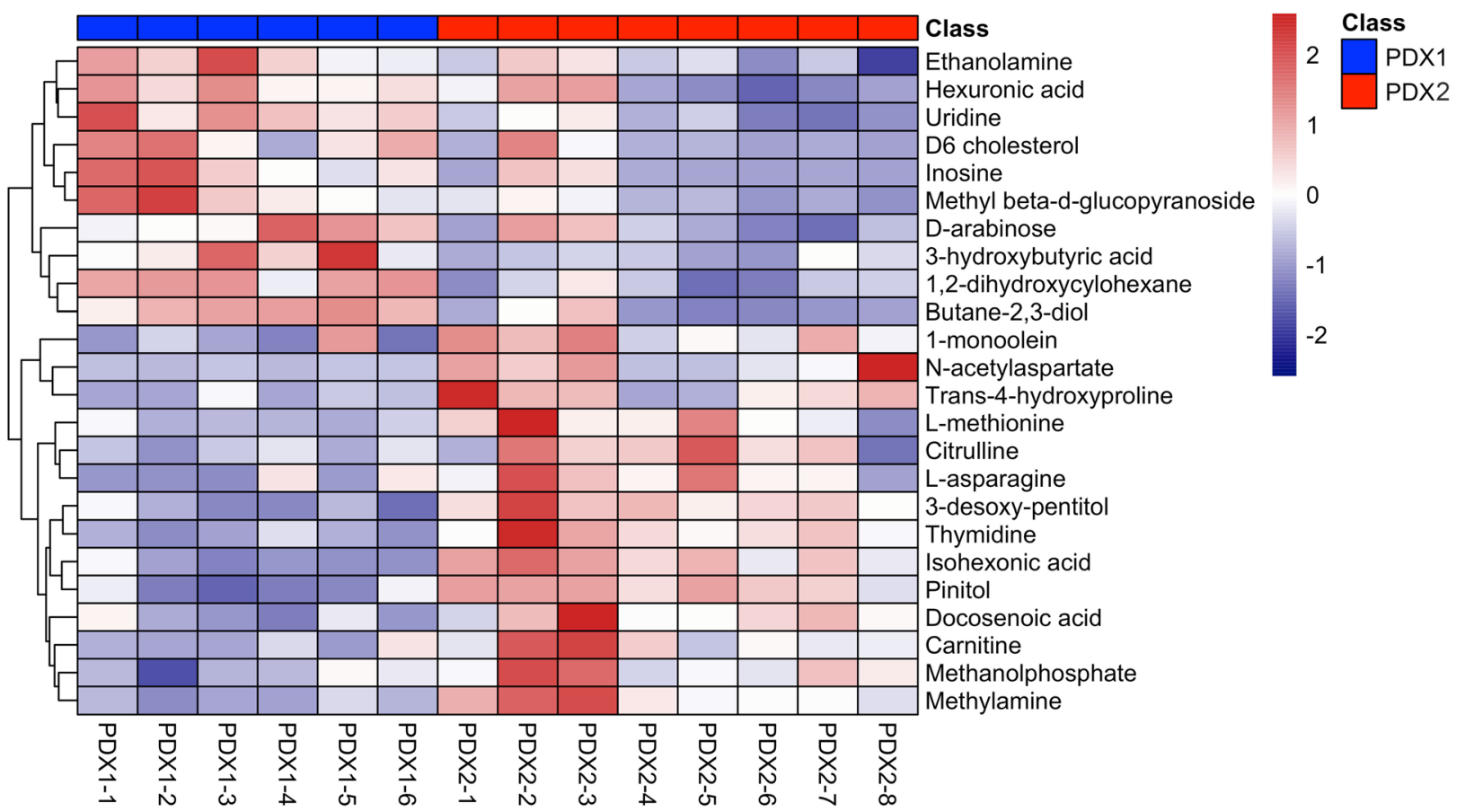

Fig. 7 Heatmap plots for overlapped differential metabolites from PDX1 and PDX2 (A), and comparison between PDX1 and PDX2 (B). Differential metabolites with VIP $>1.0$ and $P$-value $<0.05$ were used in plotting

yielding, growth, and progression, through overexpression of SLC7A5 and SLC1A5 [24-26]. Our results suggested MPM has same desire as other cancers for amino acids. Particularly, serum kynurenine, which is a downstream metabolite of tryptophan, increased significantly in both PDX1 and PDX2 in comparison to controls, suggesting an increased tryptophan metabolism. Some cancer research had opined immunosuppressive role of kynurenine [27]. In line, many studies documented increased levels of IDO and TDO in cancers, which are two enzymes catalyzing anabolism of tryptophan to kynurenine [28-30]. Collectively, dysregulated 

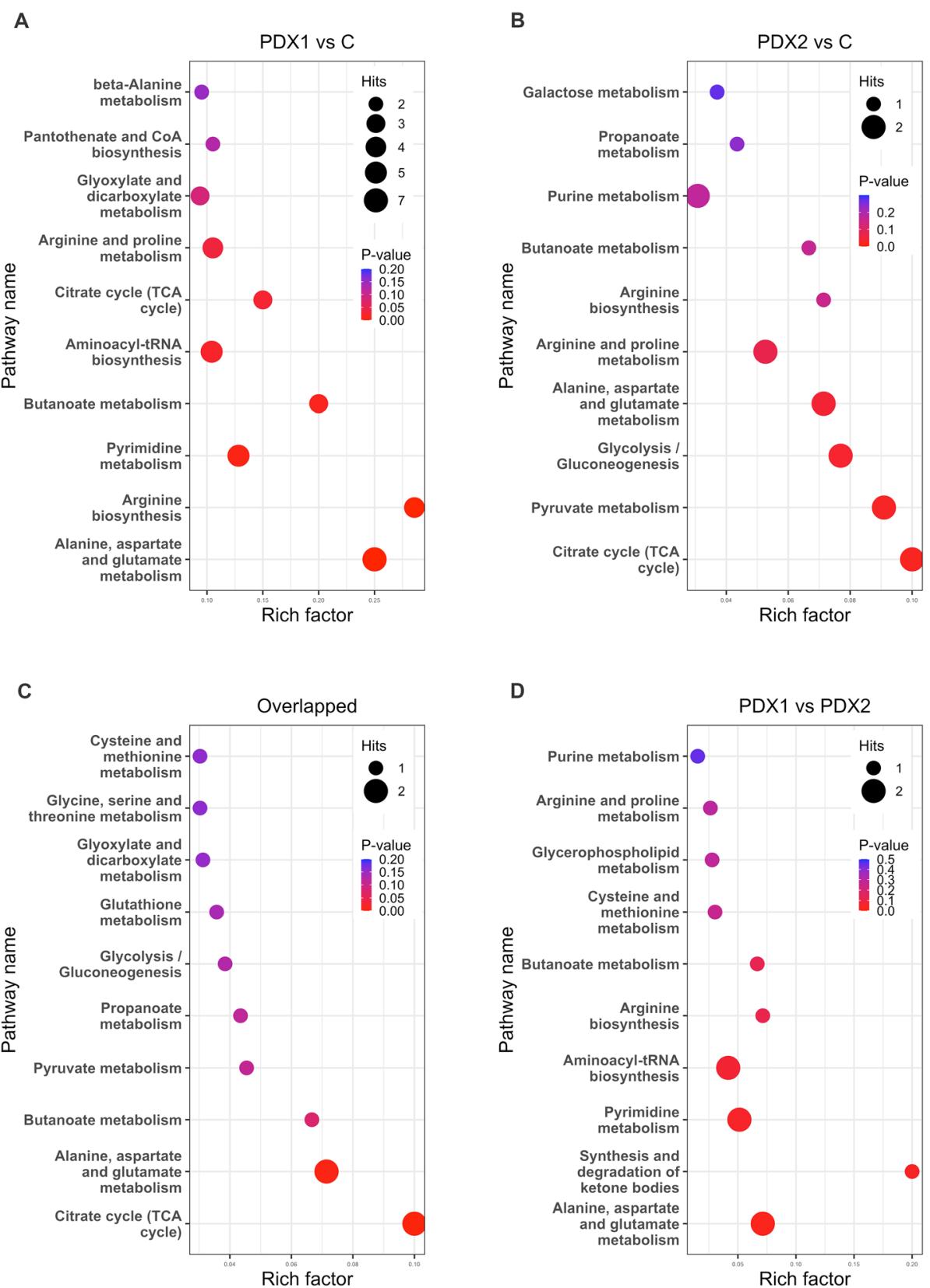

Fig. 8 Pathway enrichment results for differential metabolites from PDX1 vs. control (A), PDX2 vs. control (B), overlapped differential metabolites between PDX1 and PDX2 (C), PDX1 vs. PDX2 (D)

circulating amino acid metabolism, especially kynurenine metabolism, was a significant metabolic feature of MPM.

We also revealed dysregulations in TCA cycle and glycolysis in MPM. We detected an increase in pyruvic acid efflux, suggesting an elevated glycolysis rate. However, contradictory results were found in lactic acid, which was elevated in PDX2 but unchanged in PDX1, denoting a higher rate of aerobic glycolysis in PDX2 than PDX1. Increasing evidence has suggested that lactic acid secretion helps immune evasion of tumor by constructing a micro-environment with low $\mathrm{pH}$ that suppresses antitumor immune response [31]. Therefore, the increased glycolysis rate in MPM may have dual purposes that increase energy fueling as well as help tumor escape from immunity.

Dysregulations in metabolites of purine metabolism and pyrimidine metabolism were detected, indicating an imbalanced nucleotide metabolism. Based on our results, circulating uridine was significantly upregulated in both PDX models, which may be signs of a higher rate of 


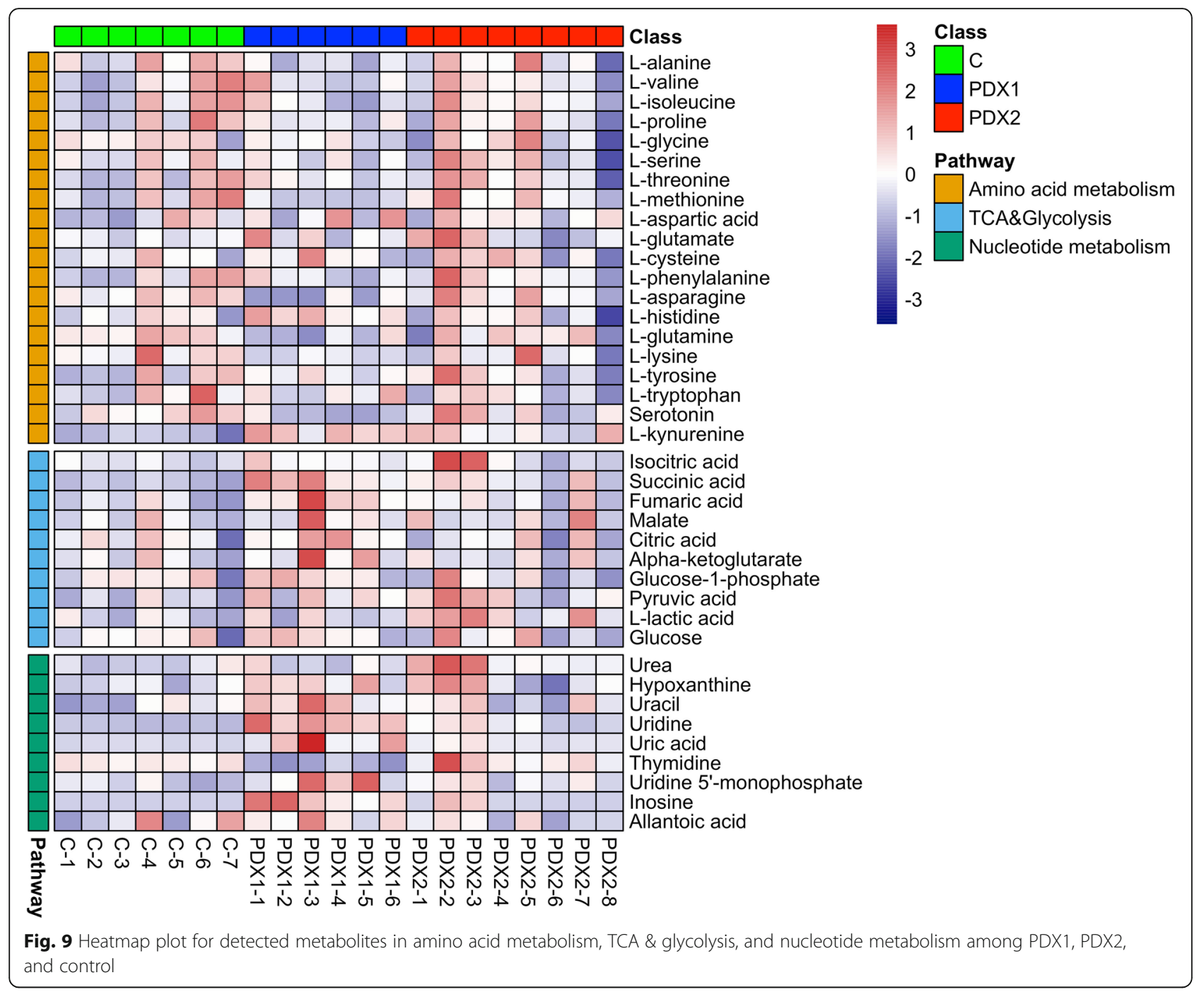

uridine synthesis in MPM. Tumor synthesizes more nucleotides, increasing deoxyribonucleic acid (DNA) and ribonucleic acid (RNA) pools that support proliferation [32]. Further we found significant elevations in uric acid, which has been reported to be released from dying tumor cells [33]. This also frequently occurs in other cancers, such as breast cancer [34], hepatocarcinoma [35], and head and neck carcinoma [36]. Uric acid also promotes tumor immune rejection [33], and serves as a pro-oxidant that induces tumor growth [37]. Herein, the excessively synthesized uridine and uric acid in MPM may be biomarkers for MPM.

However, the changed metabolites were only phenotypes which lack clear molecular mechanisms to be elucidated. Also, it is well-known that BAP1 influences metabolism in MPM, so metabolic inconsistency between samples of different BAP1 status should be compared. It is a pity that we only have one patient's information of genetic mutation, but future research with larger sample size and complete clinical information should fill in this gap.

\section{Conclusions}

In conclusion, our study developed a novel modeling technique to facilitate research in malignant mesothelioma, especially in China. In using a combination of CT scanning, pathological analysis and US-guided pleural biopsy for PDX modeling, we can remove barriers in MPM research that is caused by the scarcity in samples, thus improving availability of research in MPM or other infrequent cancers. By further coupling with metabolomics to screen for metabolic biomarkers, we can advance the current diagnostic method and treatment for MM. Nevertheless, studies with larger sample size are needed for this cancer, and molecular mechanisms as well as genetic predispositions should be further investigated to verify our results. 


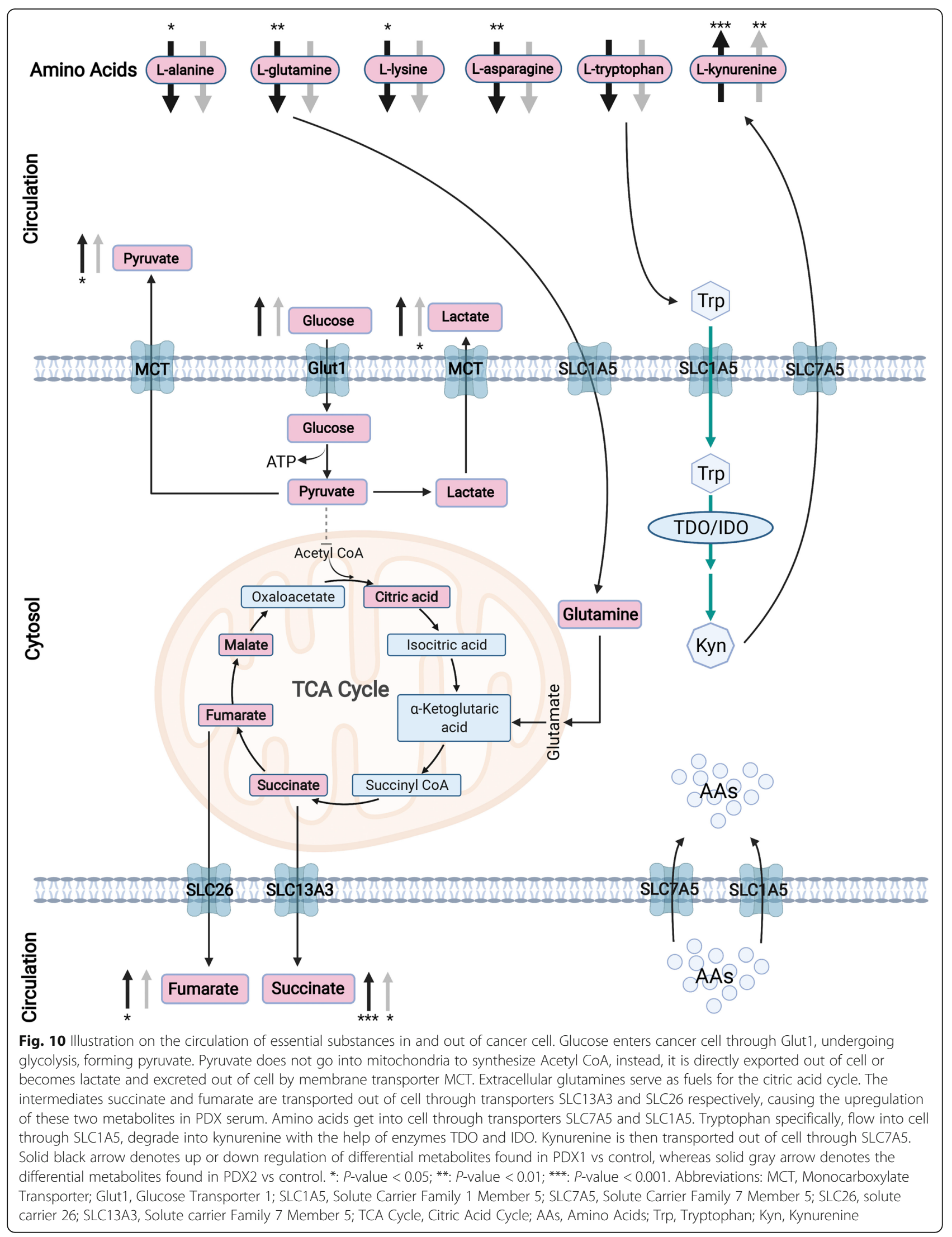




\section{Abbreviations}

MM: Malignant mesothelioma; MPM: Malignant pleural mesothelioma; PDX: Patient-derived xenograft; US: Ultrasound; CT: Computed tomography; IHC: Immunohistochemistry; GC-MS: Gas chromatography-mass spectrometry; PCA: Principal component analysis; PLS-DA: Partial least-squares-discriminant analysis; HE: Hematoxylin and Eosin; UMP: Uridine 5'-monophosphate; DNA: Deoxyribonucleic acid; RNA: Ribonucleic acid; TCA: Tricarboxylic acid; VIP: Variable importance in the projection; VATS: Video-assisted thoracoscopic surgery

\section{Supplementary Information}

The online version contains supplementary material available at https://doi. org/10.1186/s12885-021-08980-5

\section{Additional file 1. \\ Additional file 2. \\ Additional file 3. \\ Additional file 4.}

\section{Acknowledgements}

Not applicable.

\section{Authors' contributions}

Conceptualization: JL, WM \& ZC; Data curation: ZC, CY \& SS; Formal analysis: ZG \& ZC; Funding acquisition: WM, JL \& ZG; Investigation: ZC, CY \& SS; Methodology: ZC, ZG \& JL; Project administration: JL \& WM; Resources: JL, ZG \& WM; Visualization: YG \& ZC; Roles/Writing - original draft: ZC, CY \& SS; Writing - review \& editing: all authors.

\section{Funding}

This work was supported by Key R\&D Program Projects in Zhejiang Province (2018C04009); National Natural Science Foundation of China (81672315,82072577); International Cooperation Project of Zhejiang Basic Public Technology Research Program (LGJ20H010001); Projects of Zhejiang Province Medical and Health Science and Technology Plan (2017KY256); and Clinical Research Fund of Zhejiang Medical Association (2019ZYC-A77). However, they had no involvement in study design, the collection, analysis and interpretation of data, the writing of the report, and the decision to submit the paper for publication.

\section{Availability of data and materials}

All data generated or analyzed during this study are included in this published article and its supplementary information files.

\section{Declarations}

\section{Ethics approval and consent to participate}

Collection of human samples was approved by The Ethical Committee of Zhejiang Cancer Hospital (approval number: IRB-2018-9); the animal experiment was permitted by the Institutional Animal Care and Ethics Committee of Zhejiang Cancer Hospital (No. 2018-03-054).

\section{Consent for publication}

Not applicable.

\section{Competing interests}

The authors declare that they have no competing interests.

\section{Author details}

'The Cancer Research Institute, The Cancer Hospital of the University of Chinese Academy of Sciences (Zhejiang Cancer Hospital), Institute of Cancer and Basic Medicine (ICBM), Chinese Academy of Sciences, Zhejiang 310022, Hangzhou, China. ${ }^{2}$ Key Laboratory Diagnosis and Treatment Technology on Thoracic Oncology, Zhejiang 310022, Hangzhou, China.
Received: 15 June 2021 Accepted: 8 November 2021

Published online: 17 November 2021

\section{References}

1. Mao W, Zhang X, Guo Z, Gao Z, Pass HI, Yang H, et al. Association of asbestos exposure with malignant mesothelioma incidence in eastern China. JAMA Oncol. 2017;3(4):562-4. https://doi.org/10.1001/jamaoncol.2016. 5487.

2. Cao C, Tian D, Park J, Allan J, Pataky KA, Yan TD. A systematic review and meta-analysis of surgical treatments for malignant pleural mesothelioma. Lung Cancer. 2014;83(2):240-5. https://doi.org/10.1016/j.lungcan.2013.11.026.

3. Liu B, Van Gerwen M, Bonassi S, Taioli E. Epidemiology of environmental exposure and malignant mesothelioma. J Thorac Oncol. 2017;12(7):1031-45. https://doi.org/10.1016/j.jtho.2017.04.002.

4. Gray SG. Emerging avenues in immunotherapy for the management of malignant pleural mesothelioma. BMC Pulm Med. 2021;21(1):1-24. https:// doi.org/10.1186/s12890-021-01513-7.

5. Petrof O, Neyens T, Nuyts V, Nackaerts K, Nemery B, Faes C. On the impact of residential history in the spatial analysis of diseases with a long latency period: a study of mesothelioma in Belgium. Stat Med. 2020;39(26):3840-66. https://doi.org/10.1002/sim.8697.

6. Guo Z, Carbone M, Zhang X, Su D, Sun W, Lou J, et al. Improving the accuracy of mesothelioma diagnosis in China. J Thorac Oncol. 2017;12(4): 714-23. https://doi.org/10.1016/j.jtho.2016.12.006.

7. Affatato R, Mendogni P, Del Gobbo A, Ferrero S, Ricci F, Broggini M, et al. Establishment and characterization of patient-derived xenografts (PDXs) of different histology from malignant pleural mesothelioma patients. Cancers. 2020;12(12):3846. https://doi.org/10.3390/cancers12123846.

8. Wu L, Allo G, John T, Li M, Tagawa T, Opitz I, et al. Patient-derived xenograft establishment from human malignant pleural mesothelioma. Clin Cancer Res. 2017;23(4):1060-7. https://doi.org/10.1158/1078-0432.CCR-16-0844.

9. Sconfienza LM, Mauri G, Grossi F, Truini M, Serafini G, Sardanelli F, et al. Pleural and peripheral lung lesions: comparison of US-and CT-guided biopsy. Radiology. 2013;266(3):930-5. https://doi.org/10.1148/radiol.12112 077.

10. Røe OD, Anderssen E, Helge E, Pettersen CH, Olsen KS, Sandeck H, et al. Genome-wide profile of pleural mesothelioma versus parietal and visceral pleura: the emerging gene portrait of the mesothelioma phenotype. PLoS One. 2009:4(8):e6554. https://doi.org/10.1371/journal.pone.0006554.

11. Røe OD, Anderssen E, Sandeck H, Christensen T, Larsson E, Lundgren S. Malignant pleural mesothelioma: genome-wide expression patterns reflecting general resistance mechanisms and a proposal of novel targets. Lung Cancer. 2010;67(1):57-68. https://doi.org/10.1016/j.lungcan.2009.03.016.

12. Carbone M, Gaudino G, Yang H. Recent insights emerging from malignant mesothelioma genome sequencing. J Thorac Oncol. 2015;10(3):409-11. https://doi.org/10.1097/JTO.0000000000000466.

13. Fiehn O. Metabolomics - the link between genotypes and phenotypes. Funct Genomics. 2002:155-71. https://doi.org/10.1007/978-94-010-04480_11

14. Bononi A, Yang H, Giorgi C, Patergnani S, Pellegrini L, Su M, et al. Germline BAP1 mutations induce a Warburg effect. Cell Death Differ. 2017;24(10): 1694-704. https://doi.org/10.1038/cdd.2017.95.

15. Zhang Y, Shi J, Liu X, Feng L, Gong Z, Koppula P, et al. BAP1 links metabolic regulation of ferroptosis to tumour suppression. Nat Cell Biol. 2018;20(10): 1181-92. https://doi.org/10.1038/s41556-018-0178-0.

16. Zhang Y, Tang J, Zhou X, Zhou D, Wang J, Tang Q. Ultrasound-guided pleural cutting needle biopsy: accuracy and factors influencing diagnostic yield. J Thorac Dis. 2018;10(6):3244-52. https://doi.org/10.21037/jtd.2018.05. 94.

17. Zhao L, Zhang H, White JC, Chen X, Li H, Qu X, et al. Metabolomics reveals that engineered nanomaterial exposure in soil alters both soil rhizosphere metabolite profiles and maize metabolic pathways. Environ Sci Nano. 2019; 6(6):1716-27. https://doi.org/10.1039/C9EN00137A.

18. Yamamoto N, Watanabe T, Yamada K, Nakai T, Suzumura T, Sakagami K, et al. Efficacy and safety of ultrasound (US) guided percutaneous needle biopsy for peripheral lung or pleural lesion: comparison with computed tomography (CT) guided needle biopsy. J Thorac Dis. 2019;11(3):936-43. https://doi.org/10.21037/jtd.2019.01.88.

19. Jung J, Seol HS, Chang S. The generation and application of patient-derived xenograft model for cancer research. Cancer Res Treat. 2018;50(1):1-10. https://doi.org/10.4143/crt.2017.307. 
20. Zhang X, Claerhout S, Prat A, Dobrolecki LE, Petrovic I, Lai Q, et al. A renewable tissue resource of phenotypically stable, biologically and ethnically diverse, patient-derived human breast cancer xenograft models. Cancer Res. 2013;73(15):4885-97. https://doi.org/10.1158/0008-5472.CA $\mathrm{N}-12-4081$.

21. Kim MP, Evans DB, Wang H, Abbruzzese JL, Fleming JB, Gallick GE. Generation of orthotopic and heterotopic human pancreatic cancer xenografts in immunodeficient mice. Nat Protoc. 2009;4(11):1670-80 https://doi.org/10.1038/nprot.2009.171.

22. Julien $S$, Merino-Trigo A, Lacroix L, Pocard M, Goéré $D$, Mariani $P$, et al. Characterization of a large panel of patient-derived tumor xenografts representing the clinical heterogeneity of human colorectal cancer. Clin Cancer Res. 2012;18(19):5314-28. https://doi.org/10.1158/1078-0432.CCR-120372.

23. Dong X, Guan J, English JC, Flint J, Yee J, Evans K, et al. Patient-derived first generation xenografts of non-small cell lung cancers: promising tools for predicting drug responses for personalized chemotherapy. Clin Cancer Res. 2010;16(5):1442-51. https://doi.org/10.1158/1078-0432.CCR-09-2878.

24. Bodoor K, Almomani R, Alqudah M, Haddad Y, Samouri W. LAT1 (SLC7A5) overexpression in negative Her2 Group of Breast Cancer: a potential therapy target. Asian Pac J Cancer Prev. 2020;21(5):1453-8. https://doi.org/10.31 557/APJCP.2020.21.5.1453.

25. Najumudeen AK, Ceteci F, Fey SK, Hamm G, Steven RT, Hall H, et al. The amino acid transporter SLC7A5 is required for efficient growth of KRASmutant colorectal cancer. Nat Genet. 2021;53(1):16-26. https://doi.org/10.1 038/s41588-020-00753-3.

26. Alfarsi LH, El-Ansari R, Craze ML, Masisi BK, Mohammed OJ, Ellis IO, et al. CoExpression Effect of SLC7A5/SLC3A2 to Predict Response to Endocrine Therapy in Oestrogen-Receptor-Positive Breast Cancer. Int J Mol Sci. 2020; 21(4):1407. https://doi.org/10.3390/ijms21041407.

27. Chuang SC, Fanidi A, Ueland PM, Relton C, Midttun O, Vollset SE, et al. Circulating biomarkers of tryptophan and the kynurenine pathway and lung cancer risk. Cancer Epidemiol Biomark Prev. 2014;23(3):461-8. https://doi. org/10.1158/1055-9965.EPI-13-0770.

28. Prendergast GC, Malachowski WJ, Mondal A, Scherle P, Muller AJ. Indoleamine 2,3-dioxygenase and its therapeutic inhibition in Cancer. Int Rev Cell Mol Biol. 2018;336:175-203. https://doi.org/10.1016/bs.ircmb.2017. 07.004 .

29. Ye Z, Yue L, Shi J, Shao M, Wu T. Role of IDO and TDO in cancers and related diseases and the therapeutic implications. J Cancer. 2019;10(12): 2771-82. https://doi.org/10.7150/jca.31727.

30. Zhai L, Bell A, Ladomersky E, Lauing KL, Bollu L, Sosman JA, et al. Immunosuppressive IDO in Cancer: mechanisms of action, animal models, and targeting strategies. Front Immunol. 2020;11:1185. https://doi.org/10.33 89/fimmu.2020.01185.

31. Choi SYC, Collins CC, Gout PW, Wang Y. Cancer-generated lactic acid: a regulatory, immunosuppressive metabolite? J Pathol. 2013;230(4):350-5. https://doi.org/10.1002/path.4218.

32. Ariav Y, Ch'ng JH, Christofk HR, Ron-Harel N, Erez A. Targeting nucleotide metabolism as the nexus of viral infections, cancer, and the immune response. Sci Adv. 2021;7(21):eabg6165.

33. Hu D-E, Moore AM, Thomsen LL, Brindle KM. Uric acid promotes tumor immune rejection. Cancer Res. 2004;64(15):5059-62. https://doi.org/10.1158/ 0008-5472.CAN-04-1586.

34. Yue C-F, Feng P-N, Yao Z-R, Yu X-G, Lin W-b, Qian Y-M, et al. High serum uric acid concentration predicts poor survival in patients with breast cancer. Clin Chim Acta. 2017:473:160-5. https://doi.org/10.1016/i.cca.2017.08.027.

35. Lin C-c, M-c Y. B vitamins deficiency and decreased anti-oxidative state in patients with liver cancer. Eur J Nutr. 2007;46(5):293-9. https://doi.org/10.1 007/s00394-007-0665-8.

36. Dhankhar R, Dahiya K, Sharma TK, Ghalaut VS, Atri R, Kaushal V. Diagnostic significance of adenosine deaminase, uric acid and C-reactive protein levels in patients of head and neck carcinoma. Clin Lab. 2011;57(9-10):795-8.

37. Kang D-H, Ha S-K. Uric acid puzzle: dual role as anti-oxidantand pro-oxidant. Electrolytes Blood Press. 2014;12(1):1-6. https://doi.org/10.5049/EBP.2 014.12.1.1.

\section{Publisher's Note}

Springer Nature remains neutral with regard to jurisdictional claims in published maps and institutional affiliations.

\section{Ready to submit your research? Choose BMC and benefit from}

- fast, convenient online submission

- thorough peer review by experienced researchers in your field

- rapid publication on acceptance

- support for research data, including large and complex data types

- gold Open Access which fosters wider collaboration and increased citations

- maximum visibility for your research: over $100 \mathrm{M}$ website views per year

At BMC, research is always in progress.

Learn more biomedcentral.com/submissions 